\title{
Plant Diversity Development Under Planted Woody Species on Coal Mine Spoil in a Dry Tropical Environment, India: A Case Study
}

\author{
Pardeep Kumar' ${ }^{1}$, Abhishek Kumar ${ }^{1}$, Meenu Patil', Nitin K. Sharma ${ }^{2}$, Anand N. Singh ${ }^{1 *}$ \\ 1 Department of Botany, Panjab University, Chandigarh, 160014, India \\ 2 Department of Botany, Govt. College Amb, Una, HP, 177203, India \\ * Corresponding author's e-mail: dranand1212@gmail.com; ansingh@pu.ac.in
}

\begin{abstract}
The development of reconstructed woody vegetation on coal mine dumps during the trajectory of reclamation was explicitly investigated by means of a rehabilitation technique. However, limited information is available about the composition of herbaceous species during the ecosystem re-development on mine dumps. The present study attempted to assess the composition of herbaceous vegetation beneath plantation stands of four native woody species on the coal mine spoil in a dry tropical environment. After a thorough survey of the study site, a total of 44 species of herbaceous plants belonging to 14 families were recorded. Among the recorded plant families, the Poaceae contributed the highest number of species (18) across all ages of all plantation stands. The biodiversity parameter such as species richness exhibited an increasing trend with age under plantation stand of $T$. grandis only, while the rest of the plantations showed a decreasing trend. In terms of the similarity index, the plantation stands of A. procera and T. grandis were closer to each other while A. lebbeck and $D$. strictus were farthest apart. However, the highest IVI was recorded in the seedling of $A$. lebbeck under the planted stand of A. lebbeck while lowest of Rungia repens (2.85) under $A$. procera stands at $17^{\text {th }}$-year of age. In conclusion, the plantation age, dominant tree species, and species specificity have a significant impact on the development of herbaceous vegetation beneath the plantation stand of four native woody species.
\end{abstract}

Keywords: biodiversity development, coal mine spoil, herbaceous vegetation, phytosociology, rehabilitation.

\section{INTRODUCTION}

Coal is one of the main energy sources in developing countries and the expansion of industrialization needs massive energy generation for which huge quantity of coal is extracted through mining, causing extensive landscape destruction [Singh and Singh, 2006]. Quarrying for coal and minerals creates huge deposits of dumps and overburden on the unmined earth's surface. These overburdens are mostly acidic in nature, highly toxic, with poor content of nutrients [Mensah, 2015; Tripathi et al., 2016a; Singh et al., 2017; Festin et al., 2019] and compacted soil texture which reduces the draining capacity of the soil [Jha and Singh, 1992; Tripathi and Singh, 2008; Feng et al., 2019] making it unfavourable for the establishment of vegetation.
Moreover, the natural succession in the nutrient impoverished mine spoil is a slow process [Jha and Singh, 1993] with little vegetation development; therefore, artificial revegetation [Bradshaw, 1983; Leopold and Wali, 1992; Singh et al., 2004a, 2004b] and human intervention is necessary to speed up the process of rehabilitation. Hence, successful restoration of mine spoil requires the establishment of the plant species which can thrive best in nutrient-poor soil as well as improve the physico-chemical and microbial status of the soil.

In order to restore the soil fertility and amelioration of micro-climatic conditions, tree plantations are often recommended [Singh et al., 2002; Sinha et al., 2009; Mukhopadhyay et al., 2013; Ahirwal et al., 2017]. Several restoration programs by planting either native or exotic 
tree species on coal mine spoils have been successfully attempted [Chaturvedi, 1983; Parrotta, 1999; Dutta and Agrawal, 2003; Singh and Singh, 2004]. Initial colonization of mine spoils by hardy grasses and nitrogen-fixing herbaceous legumes improves the soil fertility by adding nutrients and providing aid to colonize other herbaceous species [Singh, 2004]. The rapid growth and the aggressive nature of herbaceous species can provide an important aid to check the soil erosion on the reclaimed mine sites [Franklin et al., 2012].

Herbaceous vegetation is essential for nutrient dynamics, ecological balance and modification of the prevailing conditions to some extent. It is imperative to study the herbaceous species composition and diversity of a particular area; in order to identify the key species for their conservation and restoration of the degraded environment [Jharia et al., 2013] as well as to figure out the community and ecosystem stressors. Furthermore, inventorization of the floristic elements on overburdened spoils can be helpful to predict the path of existing succession, when compared with the surrounding forest floras [Das et al., 2013].

The information on the floristic inventories of coal mined areas is very limited, particularly in India. Few studies have been carried out on naturally revegetated coal mined spoil [Deo and Panda, 2005; Singh, 2006; Ekka and Behera, 2011; Kompała-Bąba et al., 2019] but the studies dealing with floristic explorations in the plantation stands are very exiguous. Therefore, the present study was undertaken to document the herbaceous vegetation development beneath the plantations stand of four selected native woody species on coal mined spoil in a dry tropical environment, India.

\section{MATERIAL AND METHODS}

\section{Study area}

The present work was carried out at the Jayant block of Singrauli coalmine fields in the Singrauli district (the former part of the Sidhi district), Madhya Pradesh, India (Fig. 1). The Singrauli coal mines situated at (Lat. 24 $4^{\circ}$ ' 45"-

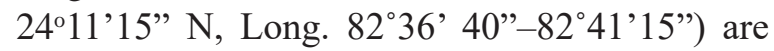
one of the largest coalfields and power complexes in the world and contribute $13 \%$ of India's coal production through opencast mining [Singh and Singh, 2004]. The climate of the area is a tropical monsoonal type with annual rainfall averages of
$1069 \mathrm{~mm}$. Annual mean temperature ranges from a minimum $6-28{ }^{\circ} \mathrm{C}$ in winter and a maximum of $20-40{ }^{\circ} \mathrm{C}$ in summer. The detailed information about the study area and type of vegetation is given elsewhere in Jha and Singh [1992]; Singh et al. [1995]; Singh and Singh [2004].

\section{Plantation stand characteristics}

The plantations stand was established by planting nursery raised seedlings of four woody species viz. Albizia lebbeck, A. procera, T. grandis, and $D$. strictus in previously dug pits of $40 \times 40 \times 40 \mathrm{~cm}$ in size at a spacing of $2 \times 2 \mathrm{~m}$. The plantations of $A$. lebbeck, A. procera, and T. grandis were raised in 1990, the former two covering an area of 1.5 ha each, while the latter - 0.5 ha. The plantation of D. strictus was raised in 1991 covering an area of 0.5 ha. The physicochemical properties of the stand were determined once in 1995 when plantations were 5 years of age and it had indicated a slightly lower bulk density, neutral pH (6.58-7.22), higher water holding capacity and poor concentration of nutrients in comparison to the fresh mine spoils [Singh et al., 2004a].

\section{Stocking density and Stand basal cover of woody plantation stand}

The number of individuals (clumps in D. strictus) in each plot was inventoried in 1995 (at $5^{\text {th }}$-year age) and after a long period in 2007 (at $17^{\text {th}}$-year of age); the existing stocking density is presented in Table 1. There was a drastic change in the stocking density of all planted woody species after such a long duration. About $50 \%$ of individuals in each planted species have declined due to competition or space problems. According to the forest department personnel (MP Ban Nigam, Sidhi), neither thinning nor pruning were done in these experimental plots. However, the stocking density at the time of plantation (1990, 1991) was 2500 individuals $h^{-1}$ for each species.

\section{Phytosociological analysis of herbaceous vegetation}

The herbaceous vegetation under plantations was analyzed through fifteen $50 \times 50 \mathrm{~cm}$ quadrats laid down in each plantation (i.e. a total of 60 quadrats for four sites at each time of sampling) at a peak time of floristic development (in the month of October 1995 and 2007, respectively). 


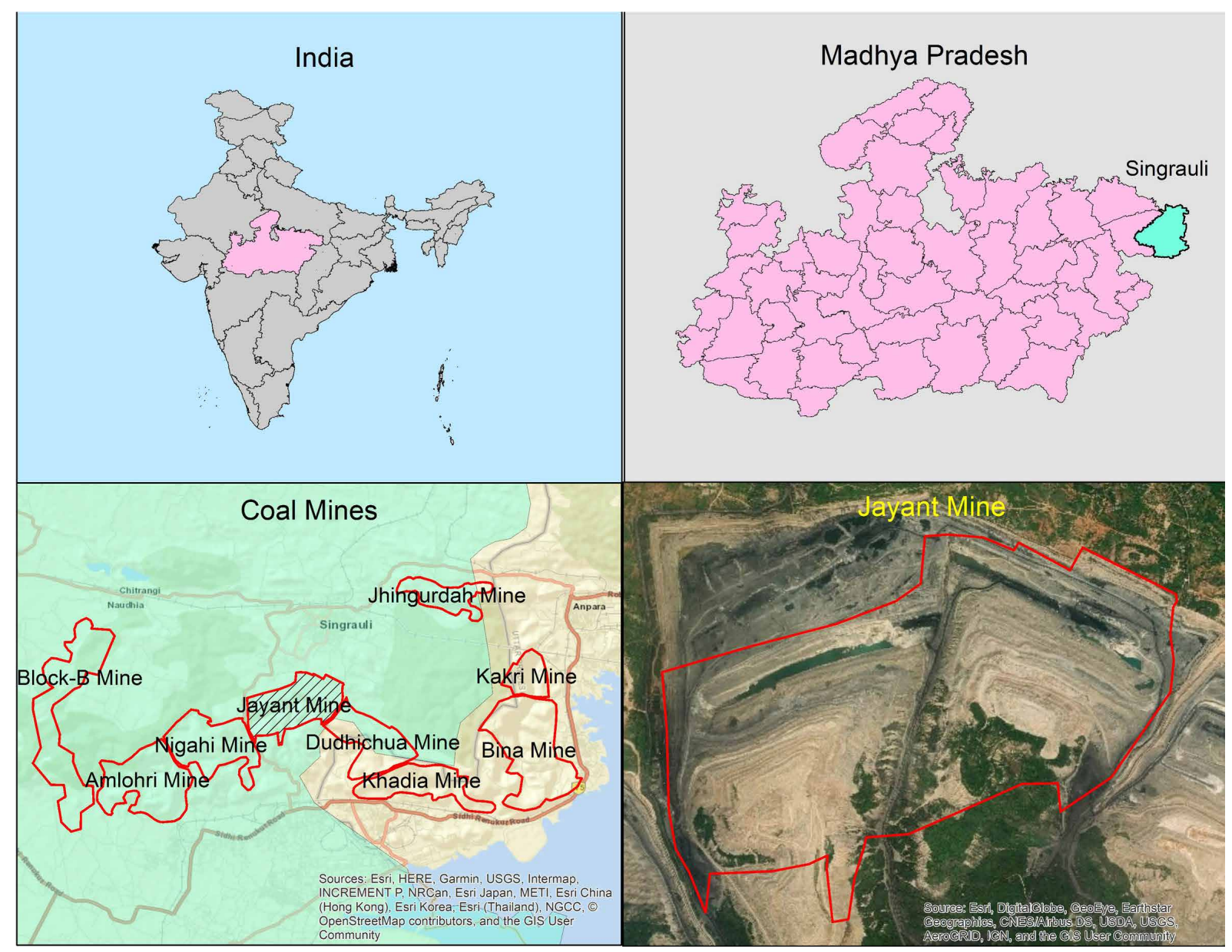

Figure 1. Map showing location of study sites

The quadrats were laid down randomly in the plantation plots; afterwards, all individuals of each quadrat were counted through tiller analysis [Singh and Yadava, 1974]. The unidentified species were recorded in the notebook, carefully collected, and stored in a modified hardboard sheet for preparing herbarium for proper identification by a well-known authority. The remaining plant samples were brought to the laboratory for further analysis. The vegetation data were quantitatively analyzed for phytosociological studies such as frequency, density, dominance, and IVI by following the method of Curtis and McIntosh [1950] and Misra [1968]:

$$
\text { Density }=\frac{\text { Total no. of individuals of a species }}{\text { Total no. of quadrats studied }}
$$

$$
\begin{gathered}
\text { Relative density }(\%)= \\
=\frac{\text { Density of a species }}{\text { Density of all the species }} \times 100
\end{gathered}
$$

$$
\begin{gathered}
\text { Frequency }= \\
=\frac{\text { Total no. of individuals of a species }}{\text { No. of quadrats in which species present }} \\
\text { Relative frequency }(\%)= \\
=\frac{\text { Frequency of a species }}{\text { Frequency of all the species }} \times 100 \\
=\frac{\text { Relative dominance }(\%)=}{\text { Biomass of all the species }} \times 100 \\
\text { IVI }=\text { Relative density }+ \text { Relative frequency }+ \\
+ \text { Relative dominance }
\end{gathered}
$$


Table 1. General characteristics such as stocking density, basal cover of planted woody species on coal mine spoil. Values (mean $\pm 1 \mathrm{SE}$ ) are means of three replicate plots. Values in the rows suffixed with different letters are significantly different with each other at $\mathrm{p}<0.05$

\begin{tabular}{|c|c|c|c|c|}
\hline \multirow{2}{*}{ Parameters } & \multicolumn{2}{|c|}{ Plantation age (year) } & \multicolumn{2}{|c|}{ Statistical values } \\
\hline & 5 & 17 & $t$ & $p$ \\
\hline \multicolumn{5}{|c|}{ Stocking density (individuals ha-1) } \\
\hline A. lebbeck & $2187 \pm 24.44^{\mathrm{a}}$ & $1817 \pm 71.44^{\mathrm{b}}$ & 10.13 & 0.010 \\
\hline A. procera & $2208 \pm 16.00^{a}$ & $1722 \pm 62.82^{b}$ & 17.73 & 0.003 \\
\hline T. grandis & $1778 \pm 117.36^{a}$ & $1434 \pm 68.38^{b}$ & 4.25 & 0.05 \\
\hline D. strictus ${ }^{*}$ & $2029 \pm 50.81^{\mathrm{a}}$ & $1469 \pm 54.00^{\mathrm{b}}$ & 18.05 & 0.003 \\
\hline \multicolumn{5}{|c|}{ Mean basal cover $\left(\mathrm{m}^{2}\right.$ individual $\left.{ }^{-1}\right)$} \\
\hline A. lebbeck & $0.006 \pm 0.0003^{a}$ & $0.023 \pm 0.002^{b}$ & -19.02 & 0.003 \\
\hline A. procera & $0.007 \pm 0.0006^{\mathrm{a}}$ & $0.022 \pm 0.004^{b}$ & -6.79 & 0.021 \\
\hline T. grandis & $0.003 \pm 0.0003^{a}$ & $0.100 \pm 0.008^{a}$ & -1.40 & 0.296 \\
\hline D. strictus ${ }^{*}$ & $0.001 \pm 0.0002^{\mathrm{a}}$ & $0.001 \pm 0.000^{a}$ & 0.58 & 0.621 \\
\hline \multicolumn{5}{|l|}{ Stand basal cover $\left(\mathrm{m}^{2} \mathrm{ha}^{-1}\right)$} \\
\hline A. lebbeck & $13.79 \pm 0.4^{\mathrm{a}}$ & $42.25 \pm 4.39^{b}$ & -43.60 & 0.001 \\
\hline A. procera & $14.98 \pm 1.36^{\mathrm{a}}$ & $37.58 \pm 7.28^{b}$ & -46.50 & 0.000 \\
\hline T. grandis & $5.81 \pm 0.58^{a}$ & $13.47 \pm 11.21^{b}$ & -35.92 & 0.001 \\
\hline D. strictus ${ }^{*}$ & $2.58 \pm 0.3^{a}$ & $1.80 \pm 0.21^{\mathrm{b}}$ & -46.84 & 0.000 \\
\hline
\end{tabular}

* Plantation age is 16-year old.

\section{Diversity indices:}

Species richness index was calculated by following the method of Margalef [1958]:

$$
\mathrm{SR}=\frac{\mathrm{S}-1}{\ln (\mathrm{N})}
$$

Index of evenness (Ew) was calzulated using the formula given by Whittaker [1972]:

$$
\mathrm{Ew}=\frac{\mathrm{S}}{\ln \mathrm{Ni}-\ln N \mathrm{~s}}
$$

Beta diversity $(\beta \mathrm{w})$ was computed as per the formula given by Whittaker [1972]:

$$
\beta=\frac{\mathrm{Sc}}{\overline{\mathrm{S}}}
$$

The similarity index between pairs of planted trees was calculated by using the IVI value similarity coefficient between the pair of planted stand vegetation types with the help of the following expression:

$$
\mathrm{S}=\frac{2 \mathrm{C}}{\mathrm{a}+\mathrm{b}} \times 100
$$

where $\mathrm{S}=$ Number of species; whereas

$\mathrm{N}=$ Total number of individuals;

$\mathrm{Ni}=$ Number of individuals of most abundant specie;
Ns = Number of individuals of least abundant species;

$\mathrm{Sc}=$ Total number of species;

$\overline{\mathrm{S}}=$ Average number of species per sample;

$\mathrm{C}=$ sum of lowest IVI of common species; "a" and "b" are the sum of IVIs of all species under both planted stands.

\section{RESULTS}

\section{Species composition}

A total of 44 species of herbaceous plants belonging to 14 families were recorded from the plantation stand (Fig. 2). The maximum number of species (23) was recorded from the $17^{\text {th }}$-yearold plantation stand of Tectona grandis (Fig. 3) while the $5^{\text {th }}$-year-old stand of Albizia lebbeck showed the lowest number of species (9) (Fig. 4).

Across all ages and all plantation stands, the Poaceae family (18) was dominant, followed by Asteraceae (11) and Fabaceae (10) families (Fig. 2). Among the 5-year-old plantations of all species, maximum RF was recorded for Hyptis suaveolens (21.21\%) under plantation stand of $A$. procera and minimum $(2.17 \%$ each) for Blumea alata, Bothriochloa pertusa, 
Corchorus capsularis, Oldenlandia corymbosa and Phyllanthus niruri under plantation stand of D. strictus. However, the corresponding parameter at the $17^{\text {th }}$-year of all plantation stand was maximum for woody seedlings of A. lebbeck (21.74\%) and minimum for Desmodium triflorum, Justicia gendarussa, Pennisetum pedicellatum $(1.16 \%$ for each) under the plantations of D. strictus (Table 2).

The relative density of the herbaceous species beneath plantations, however, reflected the same trend as shown in RF; although, the values for RD were in the range of $0.31-49.13 \%$ at $5^{\text {th }}$-year and $0.31-56.39 \%$ at $17^{\text {th }}$-year of plantation age. Moreover, the same species (Hyptis suaveolens) reflected maximum $\mathrm{RD}(49.13 \%)$ and minimum ( $0.31 \%)$ by Borreria stricta, Corchorus capsularis, Oldenlandia corymbosa and Phyllanthus niruri under $5^{\text {th }}$-year old plantation stand of D. strictus. At age of 17 , the maximum $\mathrm{RD}$ was found in the seedling of A. lebbeck (56.39\%) under A. lebbeck stand and a minimum of Rungia repens $(0.31 \%)$ under plantation stand of $A$. procera (Table 2).

However, if the seedlings of A. lebbeck been neglected, then one invader Hyptis suaveolens had highest $\mathrm{RD}$ (35.50\%) under $A$. procera plantation across all planted stands (Table 2). The relative biomass of herbaceous species across all ages and all planted stands, was maximally estimated in the seedling of $A$. lebbeck (72.80 g dry wt. basis) under the stand of A. lebbeck, while minimum of Rungia repens ( $0.14 \mathrm{~g}$ dry wt. basis) under $A$. procera stand at $17^{\text {th }}$-year (Table 2).

Although the values for RB in the present study were varied from $0.17-59.58 \mathrm{~g}$ at $5^{\text {th }}$-year, being maximum in Aristida adscensionis (59.58 g, dry wt. basis) and minimum of Cyperus compressus ( $0.17 \mathrm{~g}$ dry wt. basis) under the stand of

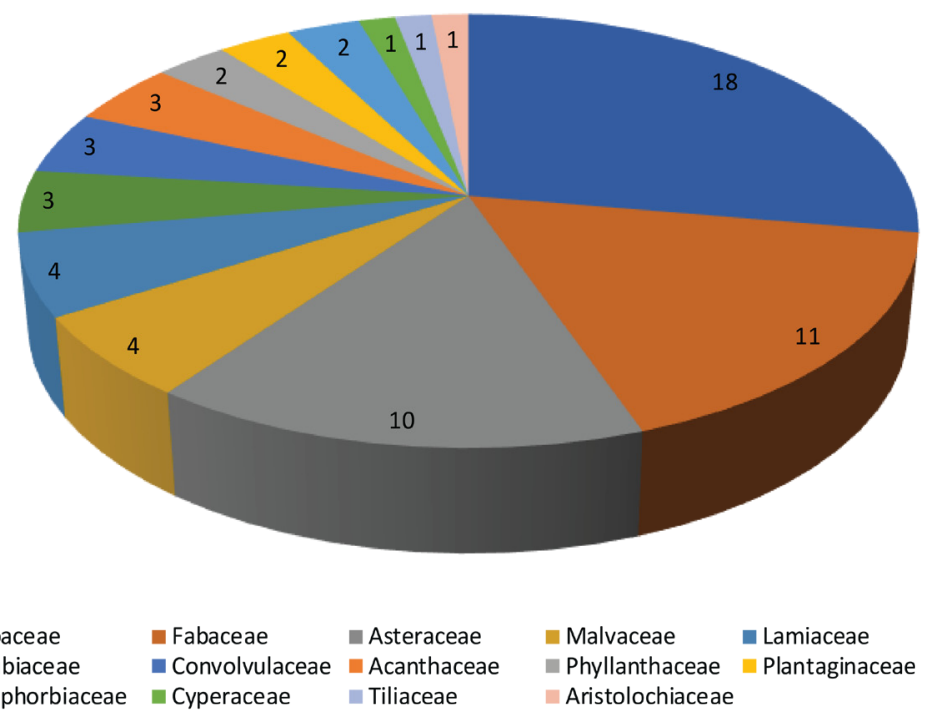

Figure 2. Family-wise number of herbaceous species recorded under all age all plantations stands of coal mine spoil

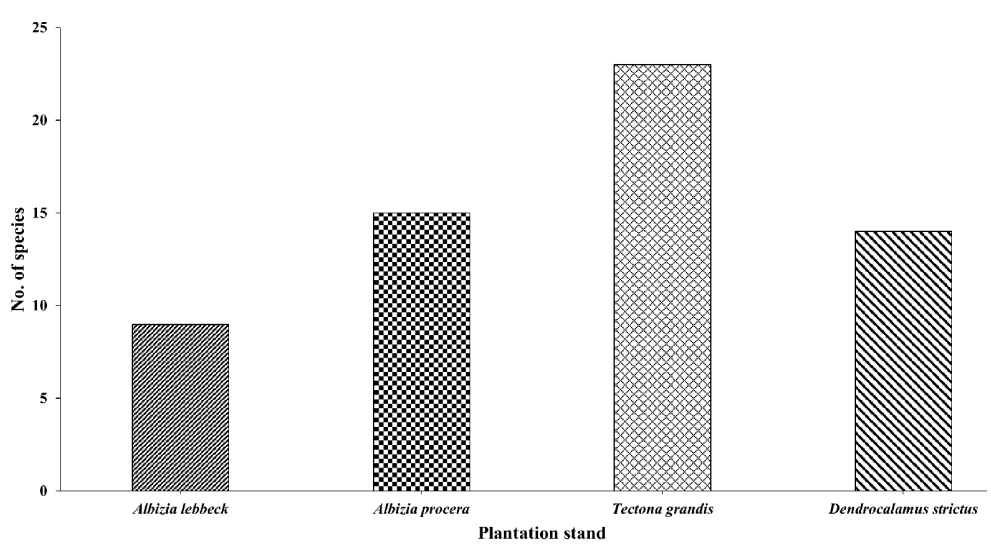

Figure 3. Total number of herbaceous species recorded under 17-year old plantation stand 


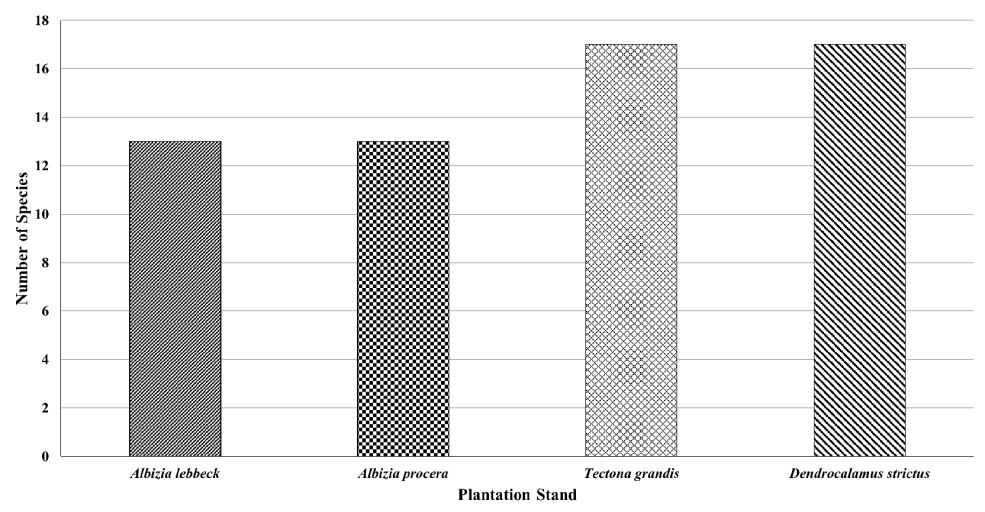

Figure 4. Total number of herbaceous species recorded under 5-year old plantation stand

A. procera plantation, the corresponding values were substantially in better range of $0.14-72.80 \mathrm{~g}$ at $17^{\text {th }}$-year of all planted stands (Table 2).

Total IVI of the herbaceous species were significantly varied in the range of 3.04-94.91 at 5 year and 2.85-150.93 at $17^{\text {th }}$-year-old plantation age of all planted species (Table 2). Across the planted stands and plantation age, the greatest IVI was recorded in the seedling of A. lebbeck under the A. lebbeck stand and lowest was of Rungia repens (2.85) under $A$. procera stand at $17^{\text {th }}$-year of age (Table 2).

\section{Species Richness, Evenness, Diversity and Similarity Index}

The species richness index of herbaceous species under planted stand varied from 2.34 (A. lebbeck) to 2.82 (D. strictus) at $5^{\text {th }}-$ and 1.21 (A. lebbeck) to 3.07 (T. grandis) at $17^{\text {th }}$-year of age. However, the variation in the values was apparently increased from $5^{\text {th }}$ - to $17^{\text {th }}$-year old plantation stands in T. grandis only, while decreased in the planted stand of A. lebbeck followed by $A$. procera and D. strictus plantation (Fig. 5). However, the effect of age, species and their interaction (age $\times$ species) on them were strongly significant (age: $\mathrm{F}_{1,16}=16.78, \mathrm{P}=0.001$; species: $\mathrm{F}_{3,16}=39.41, \mathrm{P}=0.000$ and interaction: $\mathrm{F}_{3,16}=$ 18.44, $\mathrm{P}=0.000$ ).

The species evenness is an important parameter that explains the number of individuals of the present species. A decreasing trend was observed in the present study due to the age in all planted species (Fig. 6). ANOVA indicated significantly different due to age $\left(\mathrm{F}_{1,16}=8.91, \mathrm{P}\right.$ $=0.009)$ and species $\left(\mathrm{F}_{3,16}=108.65, \mathrm{P}=0.000\right)$ but their interaction was insignificant $\left(\mathrm{F}_{3,16}=\right.$ $1.630, \mathrm{P}=0.222$ ).
Across the planted stand of all species, the alpha-diversity of herbaceous species was varied from $1.38-2.23$ at $5^{\text {th }}$ - and $1.20-2.28$ at $17^{\text {th }}$-year of plantation age, being highest in $T$. grandis under both plantation age ( $5^{\text {th }}$ - and $17^{\text {th }}$-year) and lowest in the $D$. strictus at $5^{\text {th }}$ - and $A$. lebbeck at $17^{\text {th }}$-year of stand (Fig. 7). Analysis of Variance (ANOVA) exhibited significant differences due to species $\left(\mathrm{F}_{3}\right.$, ${ }_{16}=27.20, \mathrm{P}=0.000$ ) and interaction with age (age $\times$ species $)\left(F_{3,16}=23.02, p=0.000\right)$, but the effect of age was non-significant $\left(\mathrm{F}_{3,16}=0.66, \mathrm{p}=0.430\right)$.

In turn, in this study, the $\beta$-diversity calculated between two planted stands showed insignificant differences between both plantation age $\left(5^{\text {th }}\right.$ - and $17^{\text {th }}$-year age); however, the values reflected were in the range of $1.41-1.66$ in $5^{\text {th }}$ - and $1.35-1.56$ in $17^{\text {th }}$-year of the planted stand. Among all the planted stands and age, maximum diversity was recorded in the stand of $A$. procera vs $D$. strictus in $5^{\text {th }}$-year age plantation stands and lowest in T. grandis and $D$. strictus stand at $17^{\text {th }}$-year old plantation stand (Table 3).

The similarity index of the herbaceous species under the planted stands of all selected species varied from 28.53-47.67 and 19.40-56.00, in $5^{\text {th }}$ - and $17^{\text {th }}$-year of plantation, respectively. This index was also calculated between the stands of two planted species. Although the values widely differed in between the planted stands in both ages of plantations, the highest values (47.67) under 5 years of age was reflected by T. grandis vs $D$. strictus plantation while the lowest (28.53) recorded under $A$. lebbeck vs. D. strictus plantation. Similarly, in the $17^{\text {th }}$-year of planted stand, the corresponding values were the highest (56.00) under $A$. procera vs $T$. grandis plantation and lowest (19.40) under A. lebbeck vs. A. procera plantation (Table 3). 
Table 2. Phytosociological assessment of herbaceous vegetation across different plantation stands

\begin{tabular}{|c|c|c|c|c|c|c|c|c|c|c|c|c|c|c|c|c|c|c|c|c|c|c|c|c|c|c|c|c|c|c|c|c|}
\hline \multirow{4}{*}{ Species name } & \multicolumn{8}{|c|}{ A. lebbeck } & \multicolumn{8}{|c|}{ A. procera } & \multicolumn{8}{|c|}{ Tectona grandis } & \multicolumn{8}{|c|}{ Dendrocalamus strictus } \\
\hline & \multicolumn{32}{|c|}{ Plantation age (Years) } \\
\hline & \multicolumn{4}{|c|}{$5-$} & \multicolumn{4}{|c|}{$17-$} & \multicolumn{4}{|c|}{$5-$} & & 17 & 7- & & & $5-$ & & & & 17 & 7- & & & $5-$ & & & & 16 & $6-$ & \\
\hline & $\mathrm{RD}$ & RF & $\mathrm{RB}$ & IVI & $\mathrm{RD}$ & RF & $\mathrm{RB}$ & IVI & $\mathrm{RD}$ & RF & RB & IVI & \begin{tabular}{|l|l}
$R D$ & $F$
\end{tabular} & $\mathrm{RF}$ & $\mathrm{RB}$ & IVI & $\mathrm{RD}$ & RF & $\mathrm{RB}$ & IVI & RD & $\mathrm{RF}$ & $\mathrm{RB}$ & IVI & $\mathrm{RD}$ & RF & $\mathrm{RB}$ & IVI & $\mathrm{RD}$ & $\mathrm{RF}$ & RB & $\mid \mathrm{VI}$ \\
\hline \begin{tabular}{|l|} 
Albizia lebbeck \\
(seedling)
\end{tabular} & - & - & - & -5 & 56.39 & 21.7 & 73 & 151 & - & - & - & - & - & - & - & - & - & - & - & - & - & - & - & - & - & - & - & - & - & - & - & - \\
\hline $\begin{array}{l}\text { Albizia procera } \\
\text { (seedling) }\end{array}$ & - & - & - & - & - & - & - & - & - & - & - & & \begin{tabular}{|l|l}
3.68 & 4 \\
\end{tabular} & 4.8 & 10.6 & 19.1 & - & - & - & - & - & - & - & - & - & - & - & - & - & - & - & - \\
\hline $\begin{array}{l}\text { Alysicarpus } \\
\text { monilifer }\end{array}$ & - & - & - & - & - & - & - & -1 & 11.681 & 15.25 & 5.18 & 32 & - & - & - & - & - & - & - & - & - & - & - & - & - & - & - & - & - & - & - & - \\
\hline $\begin{array}{l}\text { Aristida } \\
\text { adscensionis }\end{array}$ & - & - & - & - & - & - & - & -2 & 27.896 & 6.065 & 59.69 & 93.5 & - & - & - & -1 & 10.28 & \begin{tabular}{|l|l|}
8.98 & 7
\end{tabular} & 7.35 & \begin{tabular}{|l|l|}
26.5 & 4 \\
\end{tabular} & 4.41 & 5.4 & 4.89 & - & 4.18 & 8.59 & 8.54 & 21 & 6.5 & 10.2 & 8.26 & 25 \\
\hline $\begin{array}{l}\text { Aristolochia } \\
\text { elegans }\end{array}$ & - & - & - & - & - & - & - & - & - & - & - & - & \begin{tabular}{|l|l}
1.99 & 4 \\
\end{tabular} & $4.8:$ & 1.34 & 8.14 & - & - & - & - & - & - & -1 & 14.7 & - & - & - & - & - & - & - & - \\
\hline Blumea alata & 6.63 & 11.11 & 6.64 & 24.381 & 1.09 & 8.59 & 0.5 & $\mid 10.2$ & - & - & - & - & - & - & - & - & \begin{tabular}{|l|l}
6.56 & 6
\end{tabular} & \begin{tabular}{|l|l|}
6.74 & 1 \\
\end{tabular} & 13.6 & \begin{tabular}{|l|l|l|}
26.9 & 3 \\
\end{tabular} & 3.18 & 4.3 & 7.48 & - & 0.67 & 2.17 & 1.13 & 4 & - & - & - & - \\
\hline Borreria stricta & - & - & - & - & - & - & - & - & - & - & - & - & - & - & - & - & - & - & - & - & - & - & - & 15 & 0.31 & 2.77 & 1.13 & 4.2 & - & - & - & - \\
\hline $\begin{array}{l}\text { Bothriochloa } \\
\text { pertusa }\end{array}$ & 46 & 11.11 & 37.9 & 94.914 & 4.54 & 11.6 & 1.6 & 17.8 & - & - & - & - & 1.78 & 6 & 1.39 & 9.19 & 4.22 5 & \begin{tabular}{|l|l|l}
3 & 3
\end{tabular} & 3.49 & \begin{tabular}{|l|l|}
13.3 & 2
\end{tabular} & 2.72 & 5.4 & 1.02 & - & 2.4 & 2.17 & 3.98 & 8.6 & 10.5 & 10.2 & 10.7 & 31.3 \\
\hline Cassia tora & - & - & - & - & - & - & - & - & - & - & - & - & - & - & - & - & - & - & - & -4 & 4.013 & 3.8 & \begin{tabular}{|l|l}
2.67 & 9
\end{tabular} & 9.14 & - & - & - & - & 1.28 & 2.06 & 0.77 & 4.11 \\
\hline Chloris barbata & - & - & - & - & - & - & - & - & - & - & - & - & - & - & - & - & 2.543 & \begin{tabular}{|l|l|}
3.37 & 2 \\
\end{tabular} & 2.87 & 8.78 & - & - & -1 & 10.5 & - & - & - & - & - & - & - & - \\
\hline Clitoria ternatea & 7.81 & 11.11 & 6.08 & 251 & 1.09 & 5.79 & 0.7 & 7.568 & \begin{tabular}{|l|l}
8.04 & 1 \\
\end{tabular} & 12.11 & 1.292 & $21.5 \mathrm{C}$ & \begin{tabular}{|l|l|l}
0.63 & 3 \\
\end{tabular} & 3.6 & 0.2 & 4.44 & 16.31 & \begin{tabular}{|l|l|}
13.5 & 2 \\
\end{tabular} & 20.1 & 49.95 & 5.15 & 7 & 7.19 & - & 5.9 .1 & 10.9 & 5.8 & 23 & 11.8 & 8.16 & 7.19 & 27.2 \\
\hline $\begin{array}{l}\text { Corchorus } \\
\text { conniveans }\end{array}$ & - & - & - & - & - & - & - & - & - & - & - & - & - & - & - & - & - & - & - & - & - & - & -1 & 19.4 & 0.31 & $2.17 \mid$ & 0.79 & 3.3 & - & - & - & - \\
\hline $\begin{array}{l}\text { Cynodon } \\
\text { dactylon }\end{array}$ & 5.99 & 2.77 & 1.69 & 10.45 & - & - & - & - & - & - & - & - & - & - & - & - & - & - & - & - & - & - & - & - & - & - & - & - & - & - & - & - \\
\hline $\begin{array}{l}\text { Cyperus } \\
\text { compressus }\end{array}$ & - & - & - & - & - & - & - & -0 & 0.66 & $3.02 \mathrm{C}$ & 0.17 & 3.85 & - & - & - & - & - & - & - & - & - & - & - & - & - & - & - & - & - & - & - & - \\
\hline $\begin{array}{l}\text { Desmodium } \\
\text { triflorum }\end{array}$ & 3.63 & 8.33 & 2.4 & 14.361 & 1.65 & 10.1 & 0.5 & 12.31 & 11.689 & $9.09 \mathrm{~g}$ & 9.933 & 30.7 & 1.78 & 6 & 2.83 & 10.6 & \begin{tabular}{|l|l}
7.83 & 4 \\
\end{tabular} & 4.496 & 6.86 & 19.2 & \begin{tabular}{|l|l}
5.6 \\
2
\end{tabular} & 4.9 & 5.46 & - & 0.67 & 4.34 & 5.69 & 11 & 6.5 & 1.16 & 9.4 & 17.1 \\
\hline $\begin{array}{l}\text { Dichanthium } \\
\text { annulatum }\end{array}$ & - & - & - & - & - & - & - & - & - & - & - & - & - & - & - & -3 & 3.365 & \begin{tabular}{|l|l|l|l}
5.61 & 2
\end{tabular} & 2.87 & \begin{tabular}{|l|l|}
11.8 & 1
\end{tabular} & 1.13 & 3.8 & \begin{tabular}{|l|l|}
1.23 & 1
\end{tabular} & 15.9 & 5.22 & 4.34 & 8.42 & 18 & 8.48 & 8.16 & 7.97 & 24.6 \\
\hline $\begin{array}{l}\text { Digitaria } \\
\text { setigera }\end{array}$ & - & - & - & - & - & - & - & - & - & - & - & - & 3.89 & 6 & 4.52 & 14.4 & \begin{tabular}{|c|}
3.17 \\
\end{tabular} & \begin{tabular}{|l|l|l}
4.49 & 3
\end{tabular} & 3.24 & \begin{tabular}{|l|l|}
10.9 & 2
\end{tabular} & 2.72 & 3.8 & $2.96 \mid 6$ & 6.14 & 1.35 & 4.34 & 0.45 & 6.1 & 6.5 & 1.66 & 1.73 & 9.89 \\
\hline \begin{tabular}{|l|} 
Eragrostis \\
tenella
\end{tabular} & - & - & - & - & - & - & - & - & - & - & - & - & - & - & - & - & - & - & - & -1 & 1.51 & 3.8 & 1.049 & 9.46 & - & - & - & - & - & - & - & - \\
\hline Euphorbia hirta & 1.18 & 5.55 & 0.84 & 7.57 & - & - & - & -1 & \begin{tabular}{|l|l|l}
1.436 &
\end{tabular} & 6.061 & 1.729 & 9.21 & - & - & - & -1 & 1.465 & \begin{tabular}{|l|l}
5.61 & 1 \\
\end{tabular} & 1.39 & 8.46 & - & - & -6 & 6.33 & 2.77 & 6.52 & 4.78 & 14 & - & - & - & - \\
\hline $\begin{array}{l}\text { Euphorbia } \\
\text { tirucalli }\end{array}$ & - & - & - & - & - & - & - & - & - & - & - & - & - & - & - & - & $\mid 11.6$ & \begin{tabular}{|l|l|l|} 
& 5.74
\end{tabular} & 5.99 & 24.3 & - & - & - & - & - & - & - & - & - & - & - & - \\
\hline $\begin{array}{l}\text { Evolvulus } \\
\text { alsinoides }\end{array}$ & 4.81 & 11.11 & 6.08 & 22 & 0.95 & 8.59 & 0.3 & 9.85 & - & - & - & - & - & - & - & - & - & - & - & -1 & 1.66 & 3.2 & 3.69 & - & 0.67 & 4.34 & 1.7 & 6.7 & 3.94 & 6.12 & 3.99 & 14.1 \\
\hline $\begin{array}{l}\text { Glossocardia } \\
\text { bosvallea }\end{array}$ & - & - & - & - & - & - & - & - & - & - & - & - & 1.15 & 4.8 & 0.3 & 6.26 & - & - & - & - & - & - & -8 & 8.59 & - & - & - & - & - & - & - & - \\
\hline $\begin{array}{l}\text { Heteropogon } \\
\text { contortus }\end{array}$ & - & - & - & - & - & - & - & - & - & - & - & - & - & - & - & -1 & 1.48 & \begin{tabular}{|l|l|l|}
2.24 & 6
\end{tabular} & 6.16 & 9.88 & - & - & - & - & 21.2 & 10.9 & 35.2 & 67 & - & - & - & - \\
\hline $\begin{array}{l}\text { Hyptis } \\
\text { suaveolens }\end{array}$ & 10.3 & 11.11 & 13.3 & 34.665 & 5.22 & 10.1 & 1.4 & 16.82 & 21.272 & 21.2 & 4.74 & 47.23 & 35.5 & 16 & 14.1 & 65.2 & 16.11 & \begin{tabular}{|l|l|}
11.2 & 1
\end{tabular} & 12.5 & \begin{tabular}{|l|l|}
39.8 & 2 \\
\end{tabular} & 22.5 & 5.9 & 20 & - & 49.1 & 17.4 & 17.1 & 84 & 11.1 & 8.16 & 2.26 & 21.6 \\
\hline \begin{tabular}{|l|} 
Indigofera \\
linifolia
\end{tabular} & - & - & - & - & - & - & - & - & - & - & - & - & - & - & - & - & - & - & - & -0 & 0.68 & 2.7 & \begin{tabular}{|l|l|l|} 
& 4.35
\end{tabular} & 48.4 & - & - & - & - & - & - & - & - \\
\hline $\begin{array}{l}\text { Ipomoea } \\
\text { eriocarpia }\end{array}$ & - & - & - & - & - & - & - & -0 & \begin{tabular}{|l|l|l|}
0.66 & 3 \\
\end{tabular} & 3.021 & 1.72 & 5.4 & - & - & - & - & - & - & - & - & - & - & -3 & 3.73 & - & - & - & - & - & - & - & - \\
\hline $\begin{array}{l}\text { Justicia } \\
\text { gendarussa }\end{array}$ & - & - & - & - & - & - & - & - & - & - & - & - & - & - & - & - & - & - & - & - & - & - & - & - & - & - & - & - & 10.5 & 1.16 & 2.77 & 14.4 \\
\hline $\begin{array}{l}\text { Leucaena } \\
\text { leucocephala }\end{array}$ & 4.81 & 8.33 & 18.2 & 31.38 & - & - & - & - & - & - & - & - & \begin{tabular}{|l|l|l|}
3 & 3
\end{tabular} & 3.6 & 0.25 & 4.27 & - & - & - & - & - & - & - & - & - & - & - & - & - & - & - & - \\
\hline Leucas aspera & - & - & - & - & - & - & - & - & - & - & - & - & - & - & - & - & - & - & - & -3 & 3.93 & 4.3 & 2.36 & - & - & - & - & - & - & - & - & - \\
\hline $\begin{array}{l}\text { Ocimum } \\
\text { basilicum }\end{array}$ & - & - & - & - & - & - & - & - & - & - & - & - & 21 & 12 & 40.3 & 73.3 & - & - & - & -9 & 9.39 & 4.9 & \begin{tabular}{|l|l|l}
17.9 & 1
\end{tabular} & 10.6 & - & - & - & - & 1.97 & 4.06 & 2.99 & 9.02 \\
\hline $\begin{array}{l}\text { Oldenlandia } \\
\text { corymbosa }\end{array}$ & - & - & - & - & - & - & - & - & - & - & - & - & - & - & - & - & - & - & - & - & - & - & -3 & 32.2 & 0.31 & 2.17 & 1.13 & 3.6 & 1.97 & 4.08 & 3.99 & 10 \\
\hline $\begin{array}{l}\text { Oplismenus } \\
\text { baumannii }\end{array}$ & - & - & - & -2 & 27.9 & 13 & 22 & 62.5 & 5.846 & 6.06 & 2.5 & 14.42 & 23.4 & 12 & 14.8 & 50.3 & - & - & - & -1 & 14.3 & 5.9 & 6.35 & - & - & - & - & - & - & - & - & - \\
\hline $\begin{array}{l}\text { Panicum } \\
\text { maximum }\end{array}$ & - & - & - & - & - & - & - & - & - & - & - & - & - & - & - & - & 6.998 & \begin{tabular}{|l|l|l|}
8.25 & 3
\end{tabular} & 3.77 & 195 & 5.15 & 5.4 & \begin{tabular}{|l|l|l|}
2.97 & 2
\end{tabular} & 26.6 & - & - & - & - & - & - & - & - \\
\hline $\begin{array}{l}\text { Parthenium } \\
\text { hysterophorus }\end{array}$ & 1.80 & 2.77 & 1.69 & 6.26 & - & - & - & - & - & - & - & - & - & - & - & - & - & - & - & - & - & - & -1 & 13.5 & - & - & - & - & - & - & - & - \\
\hline $\begin{array}{l}\text { Pennisetum } \\
\text { pedicellatum }\end{array}$ & - & - & - & - & - & - & - & - & - & - & - & - & 1.67 & 6 & 0.96 & 8.65 & - & - & - & -3 & 3.25 & 3.8 & 1.5 & - & - & - & - & - & 14.4 & 1.16 & 9.38 & 24.9 \\
\hline $\begin{array}{l}\text { Phyllanthus } \\
\text { niruri }\end{array}$ & 5.99 & 8.33 & 3.45 & 17.77 & - & - & - & -0 & \begin{tabular}{|l|l|l|}
0.66 & 3 \\
\end{tabular} & $3.02 \mathrm{C}$ & 0.173 & 3.85 & - & - & - & - & - & - & - & -1 & 1.28 & 3.8 & \begin{tabular}{|l|l|}
1.23 & 8
\end{tabular} & 8.53 & 0.31 & 2.17 & 0.56 & 3 & 4.53 & 6.12 & 4.66 & 15.3 \\
\hline Rungia repens & - & - & - & - & - & - & - & - & - & - & - & - & \begin{tabular}{|l|l|l}
0.31 & 2 \\
\end{tabular} & 2.4 & 0.14 & 2.85 & - & - & - & - & - & - & -6 & 6.29 & - & - & - & - & - & - & - & - \\
\hline Scoparia dulcis & - & - & - & - & - & - & - & - & - & - & - & - & - & - & - & $-{ }_{-1}$ & 0.632 & \begin{tabular}{|l|l|}
2.24 & 1 \\
\end{tabular} & 1.02 & 3.89 & 1.2 & 3.2 & 2.23 & - & - & - & - & - & - & - & - & - \\
\hline Sida acuta & - & - & - & - & - & - & - & -5 & \begin{tabular}{|l|l|}
5.07 & 6 \\
\end{tabular} & 6.066 & 6.04 & $17.2 \mathrm{C}$ & \begin{tabular}{|l|l|}
0.83 & 4 \\
\end{tabular} & 4.8 & 1.88 & 7.52 & - & - & - & - & - & - & -6 & 6.67 & - & - & - & - & - & - & - & - \\
\hline Sida cordata & 0.54 & 2.77 & 0.28 & 3.59 & - & - & - & - & - & - & - & - & - & - & - & - & - & - & - & -0 & 0.83 & 3.2 & 0.77 & - & - & - & - & - & - & - & - & - \\
\hline $\begin{array}{l}\text { Sonchus } \\
\text { brachyotes }\end{array}$ & - & - & - & - & - & - & - & - & - & - & - & - & - & - & - & - & 3.815 & \begin{tabular}{|l|l}
5.61 & 5
\end{tabular} & 5.13 & 14.6 & 3.1 & 4.3 & 4.73 & 4.84 & 2.77 & 8.59 & 1.25 & 13 & - & - & - & - \\
\hline $\begin{array}{l}\text { Tridax } \\
\text { procumbens }\end{array}$ & - & - & - & - & - & - & - & - & - & - & - & - & - & - & - & - & 2.095 & \begin{tabular}{|l|l|}
5.61 & 2
\end{tabular} & $2.05 \leqq$ & 9.75 & 0.9 & 3.2 & \begin{tabular}{|l|l|}
1.01 & 1
\end{tabular} & 12.2 & 1.35 & 6.52 & 2.27 & 10 & - & - & - & - \\
\hline $\begin{array}{l}\text { Urochloa } \\
\text { panicoides }\end{array}$ & - & - & - & - & - & - & - & - & \begin{tabular}{|l|l|}
2.2 & 3
\end{tabular} & $3.02 \mathrm{C}$ & \begin{tabular}{|l|l|}
0.86 & 6
\end{tabular} & 6.08 & - & - & - & - & - & - & - & - & - & - & -5 & 5.14 & - & - & - & - & - & - & - & - \\
\hline Vernonia cineria & 1.18 & 5.55 & 1.41 & \begin{tabular}{|l|l|}
8.14 & 1 \\
\end{tabular} & 1.09 & 10.1 & 0.5 & \begin{tabular}{|l|l|}
11.7 & 2 \\
\end{tabular} & \begin{tabular}{|l|l}
2.86 & 6 \\
\end{tabular} & 6.066 & 6.04 & 15 & \begin{tabular}{|l|l}
1.89 & 7 \\
\end{tabular} & 7.2 & 7.4 & 16.5 & \begin{tabular}{|l|l}
1.692 \\
\end{tabular} & 2.66 & 1.6 & \begin{tabular}{|l|l}
5.95 & 1 \\
\end{tabular} & 1.66 & 4.9 & \begin{tabular}{|l|l|l}
1.77 & 8 \\
\end{tabular} & 8.29 & - & - & - & - & - & - & - & - \\
\hline
\end{tabular}


$\square$ A. lebbeck

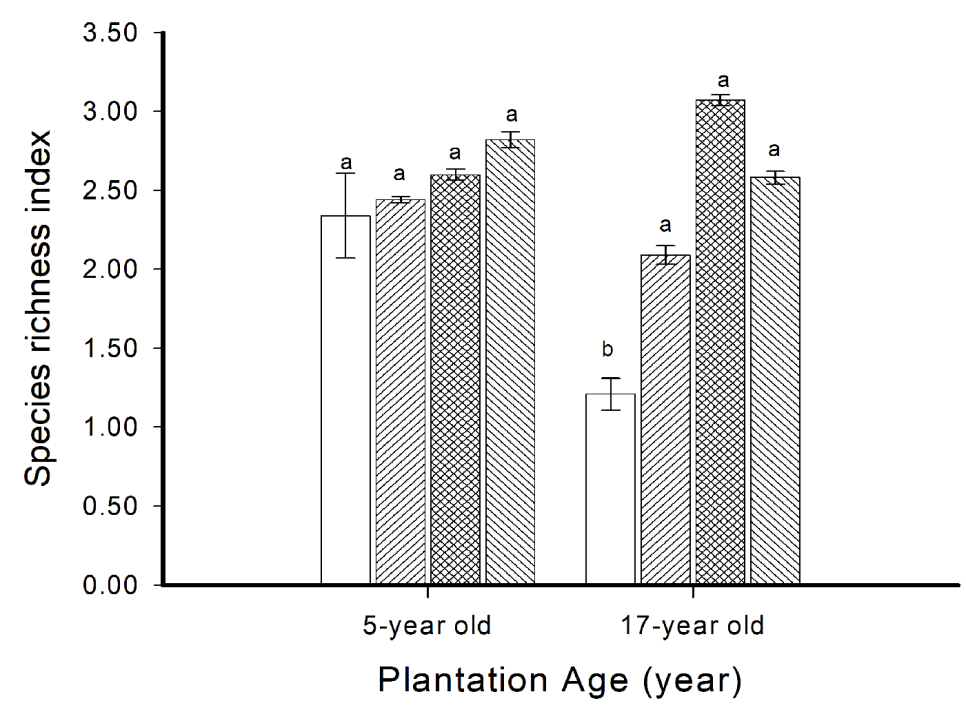

Figure 5. Species richness index of herb layer vegetation under plantations of woody species on coal mine spoil. Vertical bars are $1 \mathrm{SE}$. Values with different letters are significantly different with each other at $\mathrm{p}<0.05$

$\square$ A. lebbeck

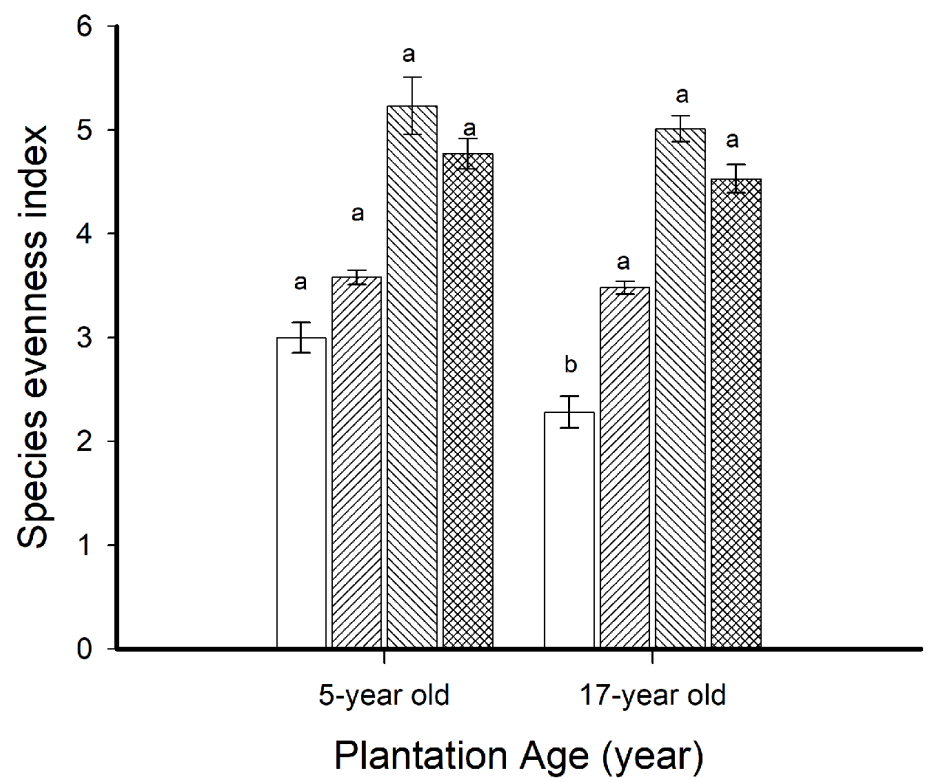

Figure 6. Species evenness of herb layer vegetation under plantations of woody species on coal mine spoil. Vertical bars are $1 \mathrm{SE}$. Values with different letters are significantly different with each other at $\mathrm{p}<0.05$

\section{DISCUSSION}

In the present study, the Poaceae family was the most commonly encountered with a maximum number of species (18) beneath all the plantation stands followed by Fabaceae (11) and Asteraceae (10) (Fig. 2). However, a similar trend was observed by several researchers from different coal mined areas [Wade and Thomspon, 1993; Ekka and Behera, 2011] in a dry tropical environment. 


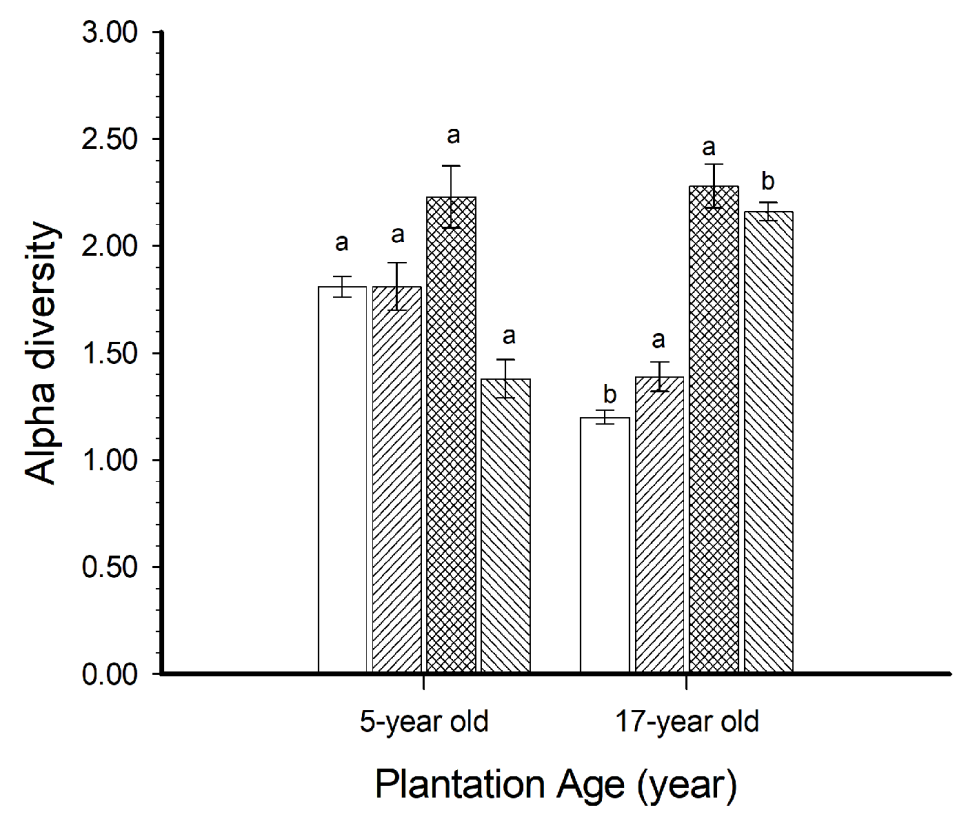

Figure 7. Alpha diversity of herb layer vegetation under plantations of woody species on coal mine spoil. Vertical bars are1SE. Values with different letters are significantly different with each other at $\mathrm{p}<0.05$

The studies conducted across European countries on afforested reclaimed sites such as [Rawlik et al., 2018 (281 sp.); Pensa et al., 2008 (100 sp.) and Mudrak et al., 2010 (79 sp.)] exhibited a relatively higher number of species as compared to the present study. However, the studies concerned with herbaceous vegetation on un-reclaimed sites such as [Hoda cova and Prach, 2003; Pensa et al., 2004; Deo and Panda, 2005; Ekka and Behera, 2011; Biswas et al., 2012] revealed a high number of species in contrary to reclaimed sites. Probably, the substrate heterogeneity [Frouz et al., 2008; Mudrak et al., 2010] and greater colonization for microhabitats
[Pensa et al., 2008; Mudrak et al., 2010] enhance the number of species on un-reclaimed sites.

A study was conducted by Singh [2012] on the same site area (19 months old naturally revegetated coal mine spoil) of the present study site revealed the occurrence of 17 herbaceous species. Limited entry of plant species in this study might be due to the slow rate of succession at young age of mined spoil, and the nature of growing tree species itself demanded a greater quantity of soil organic matter for further growth and development. However, in our study, a close taxonomic similarity was observed with the above-cited studies indicated

Table 3. Beta diversity index and Similarity index of herbaceous species under different plantation age of four native woody species on coal mine spoil. Values in the rows suffixed with different letters are significantly different with each other at $\mathrm{p}<0.05$

\begin{tabular}{|c|c|c|c|c|}
\hline \multirow{3}{*}{ Plantation stand } & \multicolumn{2}{|c|}{ Beta diversity index } & \multicolumn{2}{|c|}{ Similarity index } \\
\hline & \multicolumn{4}{|c|}{ Plantation age (Year) } \\
\hline & 5- & 17- & 5- & 17- \\
\hline A. lebbeck vs. A. procera & $1.46^{a}$ & $1.50^{\mathrm{a}}$ & $31.72^{\mathrm{a}}$ & $19.40^{\mathrm{b}}$ \\
\hline A. lebbeck vs. T. grandis & $1.60^{\mathrm{a}}$ & $1.50^{\mathrm{a}}$ & $37.17^{\mathrm{a}}$ & $33.18^{a}$ \\
\hline A. lebbeck vs. D. strictus* & $1.53^{a}$ & $1.44^{\mathrm{a}}$ & $28.53^{a}$ & $22.47^{a}$ \\
\hline A. procera vs. T. grandis & $1.60^{\mathrm{a}}$ & $1.47^{\mathrm{a}}$ & $40.69^{a}$ & $56.00^{a}$ \\
\hline A. procera vs. D. strictus ${ }^{\star}$ & $1.66^{a}$ & $1.56^{\mathrm{a}}$ & $31.34^{\mathrm{a}}$ & $24.14^{a}$ \\
\hline T. grandis vs. D. strictus ${ }^{*}$ & $1.41^{\mathrm{a}}$ & $1.35^{\mathrm{a}}$ & $47.67^{\mathrm{a}}$ & $47.76^{a}$ \\
\hline
\end{tabular}


that most of the taxon might be more or less in similar families [Holocomb and Bickford, 1952; Kozlowski,1962; Leary et al., 1997 and Goel and Behl, 2004].

However, the studies from the European coal mined areas [Mudrak et al., 2010; Pensa et al., 2008 and Rawlik et al., 2018] reported that the herb layer flora hardly overlaps within the same geographic regions. Perhaps, the parent material, geology, and microclimatic conditions were favorable to the growth of more diverse vegetation in the plantation stands. Generally, low-temperature regime and shorter period of sunny days in the European climate which is absolutely temperate in nature act as a barrier to them.

In the present study, on the basis of IVI ( $>50$ ), several species which existed in $5^{\text {th }}$ - and $17^{\text {th }}$-year-old planted stand of all species were: Bothriochloa pertusa, Aristida adscensionis, Hyptis suaveolens, Ocimum basilicum, Oplismenus burmannii, Heteropogon contortus, Clitoria ternatea and seedlings of A. lebbeck. This occurrence under both planted ages of all species stands presumably depends upon the special characters of that herbaceous species. According to Bradshaw [1983] only those species which are adapted to the special conditions of the sites, or those species which can acclimatize to the extreme conditions of the sites, are selected in a very distinctive flora on drastically disturbed lands. Predominance of grasses in young spoil age indicated the colonizing and thriving ability in a nutrient impoverished environment [Ekka and Behera, 2011].

Grasses possess a network of the fibrous root system, which efficiently binds soil particles, slows down soil erosion, and retains the soil moisture level [Helm, 1995; Woźniak, 2010; Błońska et al., 2019]. Jha and Singh [1991] reported the mechanism of natural succession of coal mine spoil. This study was conducted on the series of mine spoil age (5-, 10-, 12-, 16- and 20-year old) near to the present research sites. They found that Aristida adscensionis was a highly successful grass species for the natural succession of coal mine spoil since from the earlier stage to late-successional stage. Another grass species Bothriochloa pertusa was one of the main successional species at 20-yearold coal mine spoil exhibited better participation for the community development.

However, in the present study, both species occurred in all planted stands but do not possess the ability to dominate the community structure. Thus, out of the total recorded species amongst plantation stand, only three species (Hyptis suaveolens, Clitoria ternatea and Desmodim triflorum) were common and dominated in the all planted species, in both years of plantation age.

It appears that the biodiversity development (ground vegetation) in a rehabilitated coal mine spoil by desirable plant species is also one of the regulating factors. However, in the case of plant diversity development beneath, both planted stands were consistently similar (13 for A. lebbeck and 13 for $A$. procera) at $5^{\text {th }}$-year age followed by $D$. strictus (17) and T. grandis (17) plantation (Fig. 4). Since, Albizia lebbeck and A. procera both planted woody species are the member of same family (Fabaceae); therefore, many general characters might be similar but not all, because, A. lebbeck had comparatively better restoration potential than A. procera [Singh et al. 2004b]. However, the biodiversity development, at age $17^{\text {th }}$ of plantation stand, colonizing species under corresponding plantation were in the increasing order only in T. grandis (23) and A. procera (15) while decreasing in D. strictus (14) and A. lebbeck (9) from earlier plantation age ( $5^{\text {th }}$-year) (Fig. 3 ).

Perhaps, due to the sprouting high density of seedlings at the ground surface in the A. lebbeck plantation changed the pattern of herbaceous development in this study. However, this situation was also a trajectory in the A. procera stand due to the emergence of the own seedlings at ground level but the situation was quite better because the distribution of seedlings was sparse as compared to the plantation stand of A. lebbeck. Therefore, the situation was somewhat better in the A. procera plantation and thus permitted higher number of herbaceous species underground surface from earlier plantation age.

The basal area (BA) of plantation stand in this study, however, increased with the plantation age in all planted stands; therefore, the effect of this parameter on the biodiversity development coincided significantly with the plantation age increases. However, the relationship between the stand basal cover and alpha-diversity was not promoting to each other; therefore, the relationship was not significant $(\mathrm{p}>0.1 \mathrm{r}=0.37$; Fig. 8a) at 5-year age, but negatively correlated $(\mathrm{p}<0.01$, $r=0.72$; Fig. $8 b$ ) in the $17^{\text {th }}$-year old plantation stand of all planted species.

Perhaps, an increased basal area in the plantation stands negatively affects the entrance and development of herbaceous vegetation as well as animal diversity, at least in the developing young 
forests. In conformity with our study, Harringoton and Ewel [1997] found a declining diversity of underground species under 26-32-year-old plantation stands of Flindersia brayleyana, Eucalyptus saligna and Fraxinus uhdei at Hawaii, USA. Further, they reported a variable range of species richness (36, 42 and 39, respectively) under the same plantations. However, a lower range was found in the present study. Perhaps, in coal mine spoils, low concentration of nutrients and spoiled seed banks cannot attract a variety of vegetation in the young period [Singh and Singh, 2006].

The diversity of a developing community depends upon the species richness and their evenness. Negative significant relationships between the stand basal area with species richness were found at $5^{\text {th }}$-year $(\mathrm{p}<0.05, \mathrm{r}=0.66$; Fig. 9a) and $17^{\text {th }}$-year $(\mathrm{p}<0.01, \mathrm{r}=0.75$; Fig. $9 \mathrm{~b})$ of all planted species. However, when these data were
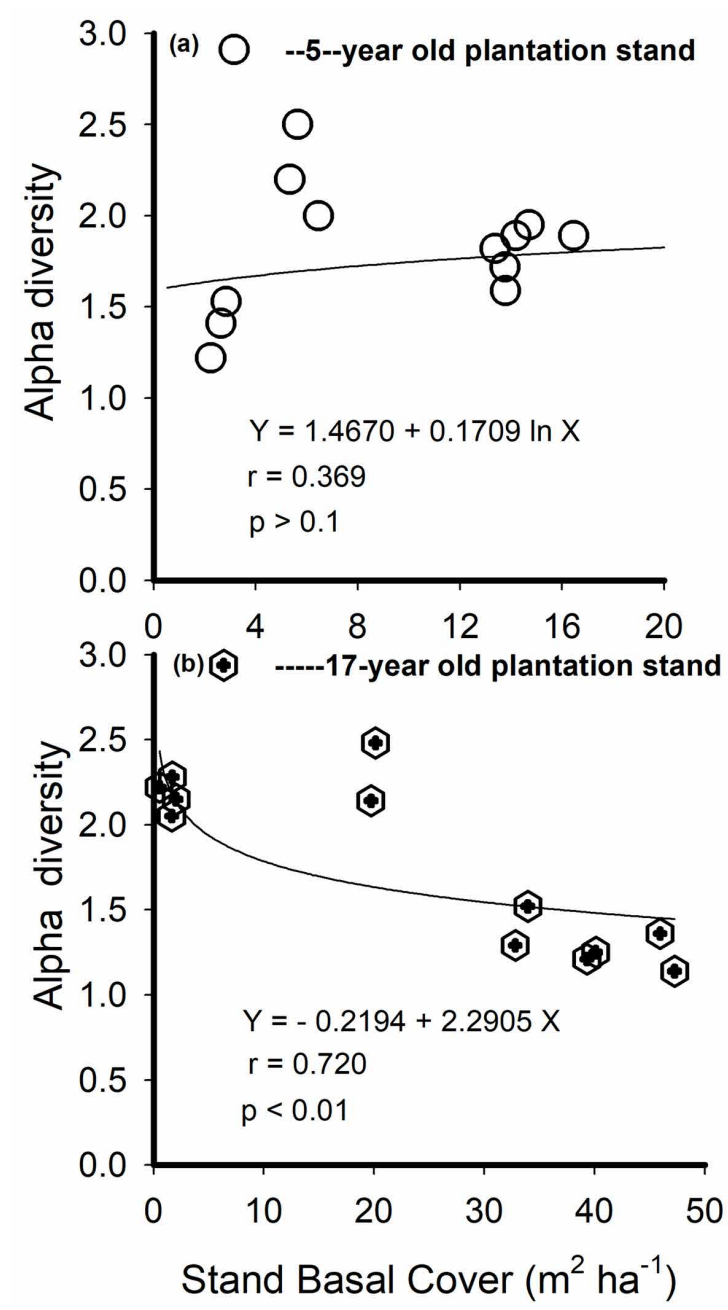

Figure 8. Relationship between stand basal cover with alpha- diversity under $5^{\text {th }}$-year old (A) and 17th-year old (B) plantations of all species on coal mine spoil tpooled across all ages and all planted species for developing the same relationship, no relationship was developed. Perhaps, it might be due to the range of species richness recorded at $17^{\text {th }}$-year old plantation stands of all species which showed a contrasting pattern.

Except T. grandis, other plantation stands (A. lebbeck $>$ A. procera $>D$. strictus) showed a slightly declining trend in species richness; furthermore, poor space and high competition between individuals for resources [Wilson, 1988; Spies and Franklin, 1991; Bingham et al.,1991; Jefferson, 2004] constrained the pace of vegetation development. Moreover, the plantation plots of $A$. lebbeck and A. procera at $17^{\text {th }}$-year of age showed the self-regenerating efficiency which reduces the entry of other herbaceous species on the ground surface due to a profuse number of seedlings. In conformity with this, Parrotta [1992] and

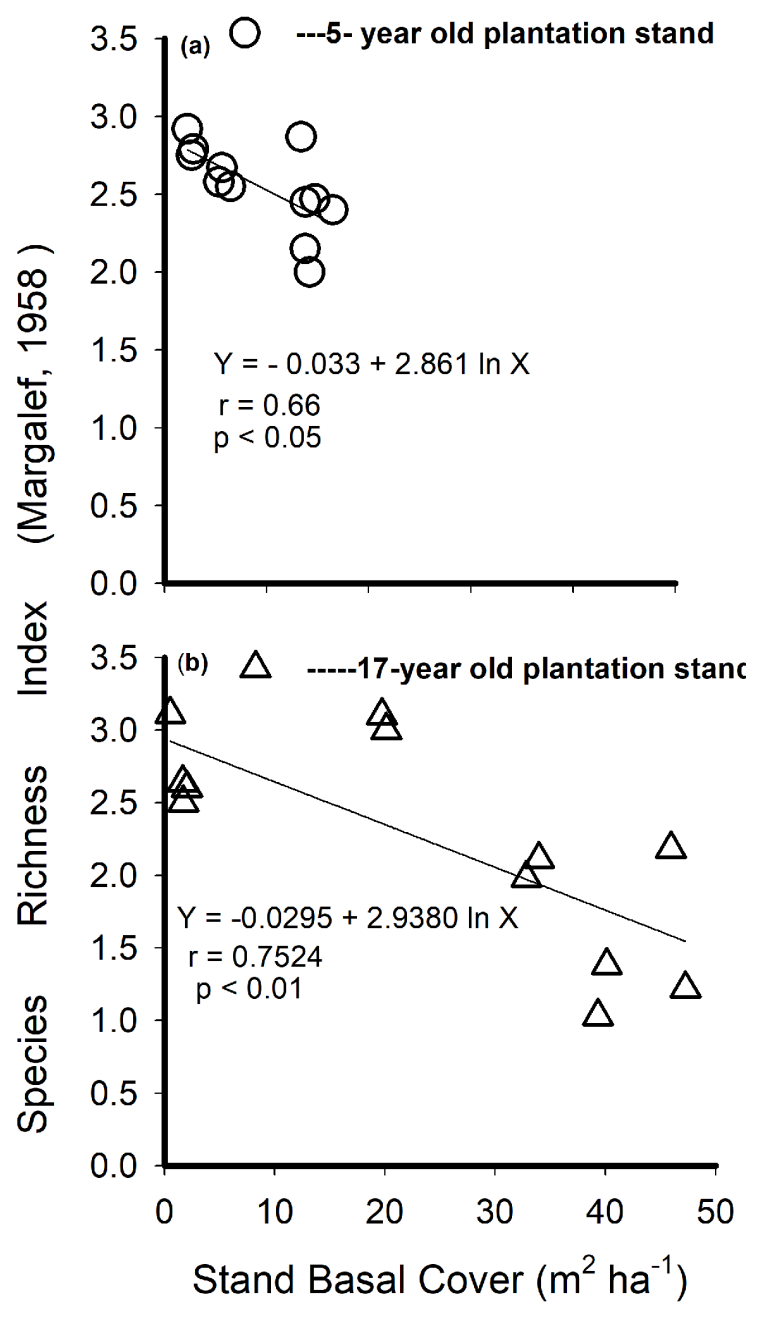

Figure 9. Relationship between stand basal cover of all planted woody species with species richness of herbaceous species at $5^{\text {th }}$-year old (A) and $17^{\text {th }}$-year old (B) plantation stand on coal mine spoil 
Keenan et al. [1997] reported the same trend in their study.

In the present study, however, the effect of stand basal area on species evenness was also found. A strong negative relationship between the stand basal area with species evenness index for $5^{\text {th }}$-year (p $<0.001, r=0.84$; Fig. $10 \mathrm{a}$ ) and $17^{\text {th }}$-year of age of all planted species $(\mathrm{p}<0.001, \mathrm{r}=0.83$; Fig. $10 \mathrm{~b})$ was found. Perhaps, the effect of the basal area is a strongly influential factor for the colonization of a new species on the ground surface of the planted species on the coal mine spoil.

In the present study, the relationship between basal area with species richness for all species of all ages was negatively correlated $(\mathrm{p}<0.0001, \mathrm{r}=0.76$; Fig. 11a) while evenness exhibited a positive correlation $(\mathrm{p}<0.01$, $r=0.49$; Fig. 11b). Perhaps, each planted species had a specific effect on coal mine spoil; therefore, under increasing age, the plantation stands

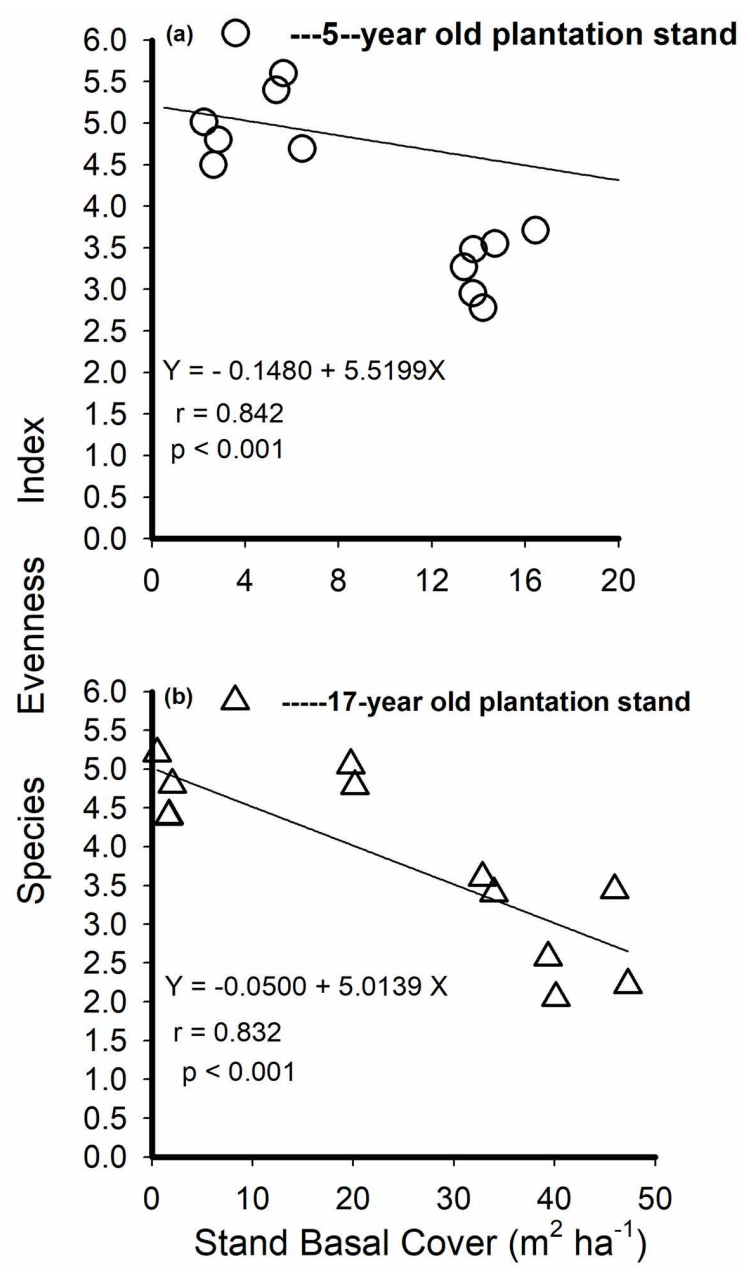

Figure 10. Relationships between stand basal cover of all planted woody species with species evenness index of herbaceous species at $5^{\text {th }}$-year old (A) and $17^{\text {th }}$-year old (B) plantation stands on coal mine spoil significantly expanded their size in terms of structural parameters such as biomass and their height, and thus, light intensity and shrinking space of ground cover might reduce a significant number of herbaceous diversity.

Additionally, heliophytes (light loving plants) would be highly restricted in the shade areas of the plantation stands. However, developing young forests on the coal mine spoil are regulated by the better qualities of the physicochemical properties of soil as reported by Singh et al. [1995] and Singh et al. [2004a, b]. A detailed study of the impacts of these plantations on soil redevelopment of the coal mine spoil in chronosequence (3-6 year) is available in Singh [1999]; Singh and Singh [1999]; Singh et al., [2004a, b]; Singh et al., [2006] and Singh and Singh [2006].

On the basis of all those corresponding reports, it was found that except $T$. grandis, the plantations of A. lebbeck, A. procera and D. strictus
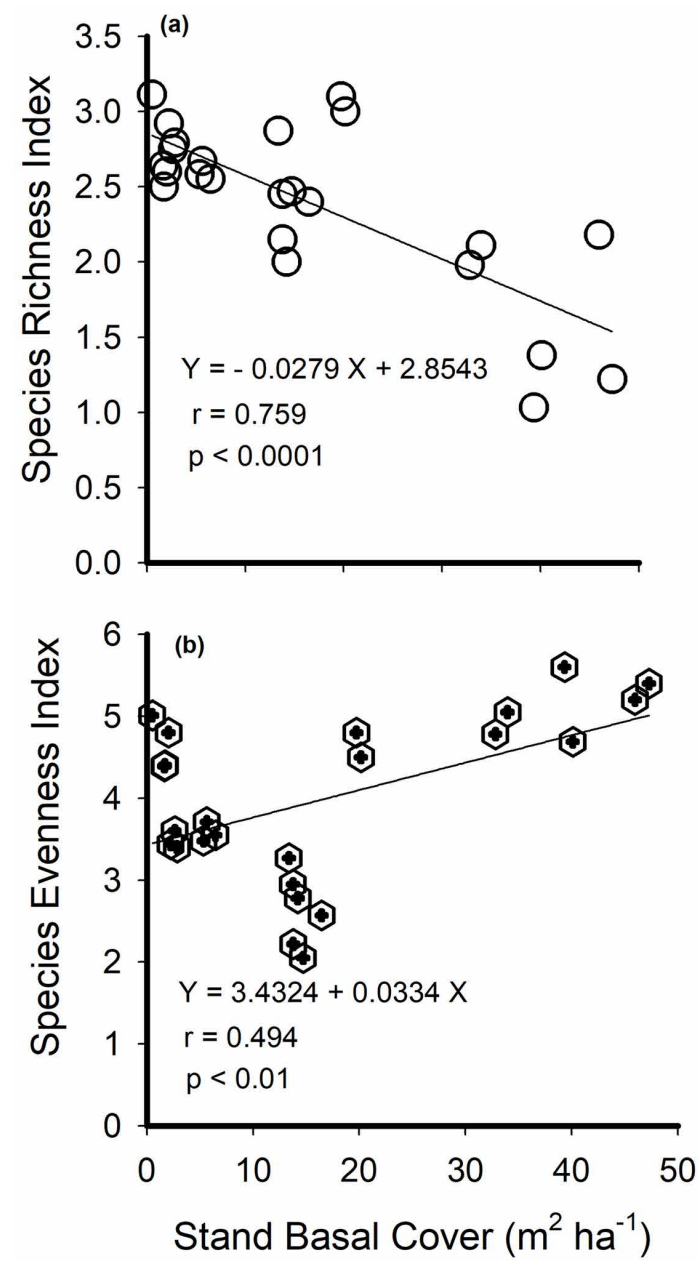

Figure 11. Relationships between stand basal cover of all planted species with species richness index (A) and species evenness index (B) of all age plantations on coal mine spoil 
are much better for the restoration of a degraded coal mine spoil. Perhaps due to that reason, the canopy cover of all these plantations was substantially developed. Contrary to this, the canopy cover of $T$. grandis plantation was less spread and not in ovate shape that provided sufficient light to the undergrowth.

The plantations of D. strictus and T. grandis are non-leguminous in nature but $D$. strictus (a member of grass family: Poaceae) exhibited surprising better efficiency of restoring the coal mine spoil at least from the initial stage of the rehabilitation [Singh and Singh, 1999] as compared to $A$. procera (a legume) and non-legume $T$. grandis. Perhaps, the plantation of $T$. grandis could not attain the restoring efficiency due to low efficiency of biomass production rates and growth behavior; however, based on the socio-economical point, it has been recommended for planting in the degraded area because of the precious and valuable timber quality.

Moreover, the biodiversity development was substantially better in this plantation stand at both planted age as compared to the other planted stands of $A$. procera, $D$. strictus and A. lebbeck suggesting that colonizing of herbaceous species under the plantation stands of a coal mine spoil was not regulated as a whole by the properties of the planted species. The species number found in these plantation stands was comparatively lower (9-23) than in the naturally vegetated coal mine spoils (19-45) under different ages at the adjoining sites of present research area. Jha and Singh [1991] indicates that the dominance of herbaceous species at the initial level of mine spoil restoration by own acclimatizing property but later on controlling by the governing system of overstory population of the plants

Wiryono and Siahaan [2013] reported lower species richness of herbaceous vegetation (17 species) under 1.5-year-old Gmelina arborea stand in Bengkulucoal mined land, Indonesia and they presumed that the lower species richness was due to the recent reclamation and low fertility of the mine soil. However, Woch et al. [2013] reported higher species richness (197 species) at Siersza hard coal mine, Poland wherein they inferred that high species richness in this small area is owing to the close proximity and ecological amplitude of a certain group of species.

Although the data concerning the restoration of coal mine spoil by natural succession is more exhaustive and available [Iverson and Wali, 1982;
Jha and Singh, 1991, 1992; Bradshaw 1997; Wali, 1999; Singh, 2004], there is insufficient amount of data on biodiversity development on coal mine spoil under planted woody species. In this research site, the parent materials, climate, rainfall or other edaphic factors are the same for all plantations. Therefore, the difference in the pattern of biodiversity development in each plantation species might be due to the nature of the specificity of species.

In agreement with this, the earlier study documented that the plantations of $A$. lebbeck and $D$. strictus showed substantially better efficiency for biomass production, deposition of nutrients $(\mathrm{C}, \mathrm{N}$, and $\mathrm{P}$ ) on the mine spoil surface by leaf litterfall followed by A. procera and T. grandis plantation [Singh et al., 2004a,b]. However, two planted species (A. lebbeck and A. procera) are leguminous in nature but their restoring efficiency is different from each other [Singh et al., 2004b].

The species diversity is an important characteristic since variations in the diversity under different plantations of the present study are presumed to be the effect of plantation species only, because, the biotic and abiotic components of this degraded ecosystem are the same for all stands. Therefore, the variations that occurred in the plantation stand for such an important parameter of biodiversity is somewhat the impact of its own plantation stand. Our finding is in agreement with some studies [Pensa et al., 2008; Mudrak et al., 2010], but it is much more difficult to predict a uniform trend for all kinds of mining habitats across globe, as nature and properties of the mine spoils may be heterogeneous and physically, chemically as well as biologically vary from one site to another site.

Thus, in the case of $T$. grandis plantation, the species richness was increased from $5^{\text {th }}$ - to $17^{\text {th }}$-year of age, while in other plantations, a decreasing trend appeared with plantation age indicating the species-specific tendencies. On the other hand, the space problems in the stands would be one of the key barriers for new species colonizing plantations [Jha et al., 1999].

\section{CONCLUSION}

The information regarding the reconstruction of biodiversity development under the planted woody species on coal mine spoil is scanty. In the present study, 44 species of herbaceous plants belonging to 14 families were recorder. In the 
$17^{\text {th }}$-year old plantation stand of $T$. grandis, the highest number of species was exhibited while the development of plant diversity under A. lebbeck was not pronounced. Across all ages of plantation stands, the Poaceae family was more dominant and contributed to the maximum allocation in the community development on ground surface. A declining trend was observed in the species richness in all the plantation stands except $T$. grandis, as the age increases.The environmental conditions were the same for all the plantation stands; thus, the fluctuation in herbaceous vegetation is the impact of individual species only. The competition between herbaceous species for space, nutrients, and light may constraint the community development. Since herbaceous vegetation regulates the production of organic matter, soil-biota, litter decomposition and nutrient channeling in the soil, the diversity and composition of herb layer must be taken into consideration while establishing restoration projects and making policies for the rehabilitation of mine-spoiled ecosystems.

\section{Acknowledgments}

The corresponding author ANS would like to thank Prof. J.S. Singh Emeritus Professor, Department of Botany, Banaras Hindu University, Varanasi for noble guidance during the course of doctoral study. Northern Coal India Limited (NCL) and department of forest personnel, Singrauli for providing necessary facilities and granting permission to carry out a field survey and vegetation sampling. Also, thanks to the University Grants Commission, Government of India for providing financial assistance in form of major research project (2010-2013). Thanks, are also due to the chairperson, Botany Dept., Panjab University, Chandigarh for providing necessary facilities. All of us (PK, AK, and MP) would like to acknowledge UGC, Government of India for providing financial assistance in form of Junior Research Fellowship.

\section{REFERENCES}

1. Ahirwal J., Maiti SK., Reddy MS. 2017. Development of carbon, nitrogen and phosphate stocks of reclaimed coal mine soil within 8 years after forestation with Prosopis juliflora (Sw.) Dc. Catena, 156, 42-50.

2. Bingham B.B., Sawyer J.O. 1991. Distinctive features and definitions of young, mature, and oldgrowth Douglas-fir/hardwood forests. In: Ruggiero L.F., Aubry K.B., Carey A.B., Huff M.H.(eds.) Wildlife and Vegetation of Unmanaged Douglasfir Forests. US Forest Service General Technical Report. PNW-285, Portland, 363-373.

3. Biswas C.K., Mishra S.P., Mukherjee A. 2012. Diversity and composition of vegetation on aged coalmine overburden dumps in Sonepur Bazari area, Raniganj, West Bengal. Journal of Environmental Biology, 35(1), 173.

4. Błońska A., Kompała-Bąba A., Sierka E., Bierza W., Magurno F., Besenyei L., Woźniak G. 2019. Diversity of vegetation dominated by selected grass species on coal-mine spoil heaps in terms of reclamation of post-industrial areas. Journal of Ecological Engineering 20(2), 209-217.

5. Bradshaw A.D. 1983. The reconstruction of ecosystems. Presidential Address to the British Ecological Society. Journal of Applied Ecology, 20, 1-17.

6. Bradshaw A.D. 1997. What do we mean by restoration? In: Krystyna M., Urbansk A., Nigel R., Webb., Edwards PJ. (eds.) Restoration Ecology and Sustainable Development. Cambridge University Press, Cambridge, 8-13.

7. Chaturvedi J.K. 1983. Afforestation of bauxite mined area in central India. Indian Forester, 109(7), 458-65.

8. Curtis J.T., Mclntosh R.P. 1950. The interrelations of certain analytic and synthetic phytosociological characters. Ecology, 3, 434-455.

9. Das M., Dey S., Mukherjee A. 2013. Floral succession in the open cast mining sites of Ramnagore colliery, Burdwan District, West Bengal. Indian Journal of Science Research, 4(1), 125-130.

10. Deo B, Panda P.C. 2005. Vegetation and flora of an open cast coal mined area in South Bolanda, Talcher, Orissa. Journal of Economic and Taxonomic Botany, 29(1), 22-30.

11. Dutta R.K., Agrawal M. 2003. Restoration of opencast coal mine spoil by planting exotic tree species: a case study in dry tropical region. Ecological Engineering, 21(2-3), 143-51.

12. Ekka N.J., Behera N. 2011. Species composition and diversity of vegetation developing on an age series of coal mine spoil in an open cast coal field in Orissa, India. Tropical Ecology, 52(3), 337-343.

13. Feng Y., Wang J., Bai Z., Reading L. 2019. Effects of surface coal mining and land reclamation on soil properties: A review. Earth-Science Reviews, 191, 12-25.

14. Festin E.S., Tigabu M., Chileshe M.N., Syampungani S., Odén P.C. 2019. Progresses in restoration of post-mining landscape in Africa. Journal of Forestry Research, 30(2), 381-96. 
15. Franklin J.A., Zipper C.E., Burger J.A., Skousen J.G., Jacobs D.F. 2012. Influence of herbaceous ground cover on forest restoration of eastern US coal surface mines. New Forests, 43, 905-924.

16. Frouz J., Prach K., Pižl V., Háněl L., Starý J., Tajovský K., Materna J., Balík V., Kalčík J., Řehounková K. 2008. Interactions between soil development, vegetation and soil fauna during spontaneous succession in post mining sites. European Journal of Soil Biology, 44(1), 109-21.

17. Goel V.L., Behl H.M. 2004. Productivity assessment of three leguminous species under high- density plantations on degraded soil sites. Biomass \& Bioenergy, 27, 403-409.

18. Harrington R.A., Ewel J.J. 1997. Invasibility of tree plantations by native and non-indigenous plant species in Hawaii. Forest Ecology and Management, 99, 153-162.

19. Helm D.J. 1995. Native grass cultivars for multiple revegetation goals on a proposed mine site in south central Alaska. Restoration Ecology, 20, 111-122.

20. Hodačová D, Prach K. 2003. Spoil heaps from brown coal mining: technical reclamation versus spontaneous revegetation. Restoration Ecology, 11(3), 385-91.

21. Holcomb C.J., Bickford C.A. 1952. Growth of yellow poplar and associated species in West Virginia as a guide to selective cutting. Station Paper NE-52. Upper Darby, PA: US Department of Agriculture, Forest Service, Northeast Forest Experimental Station. pp. 14.

22. Iverson L.R., Wali M.K. 1982. Reclamation of coal mined lands: The role of Kochia scoparia and other pioneers in early succession. Reclamation and Revegetation Research, 1, 123-160.

23. Jefferson L.V. 2004. Implications of plant density on the resulting community structure of mine site land. Restoration Ecology, 12 (3), 429-438.

24. Jha A.K, Singh A., Singh A.N., Singh J.S. 1999. Tree canopy development in young plantation raised on coalmine spoil affect the growth of herbaceous vegetation. Indian Forester, 125 (3), 305-307.

25. Jha A.K., Singh J.S. 1991. Spoil characteristics and vegetation development of an age series of mine spoils in a dry tropical environment. Vegetatio, 97, 63-76.

26. Jha A.K., Singh J.S. 1992. Rehabilitation of mine spoil. In: Singh J.S. (eds.). Restoration of degraded lands: Concepts and strategies. Rastogi publications, Meerut, India, 210-254.

27. Jha A.K., Singh J.S. 1993. Growth performance of certain directly seeded plants on mine spoil in a dry tropical environment, India. Indian Forester, 119(11), 920-7.

28. Jharia M.K., Bargali S.S., Swamy S.L., Oraon P.R. 2013. Herbaceous diversity in proposed mining area of rowghat in Narayanpur district of Chhattisgarh, India. Journal of Plant Development Sciences, 5(4), 385-393.

29. Keenan R., Lamb D., Woldring O., Irvine T., Jensen R. 1997. Restoration of plant biodiversity beneath tropical tree plantations in Northern, Australia. Forest Ecology and Management, 99, 117-131.

30. Kompała-Bąba A., Bierza W., Błońska A., Sierka E., Magurno F., Chmura D., Besenyei L., Radosz Ł., Woźniak G. 2019. Vegetation diversity on coal mine spoil heaps-how important is the texture of the soil substrate?. Biologia, 74(4), 419-36.

31. Kozlowski T.T. 1962. Tree Growth. The Ronald Press Co. New York, 442.

32. Leary R.A., Nimerfro K., Holdaway M., Brand G., Burk T., Kolka R., Wolf A. 1997. Height growth modelling using second order differential equations and the importance of initial height growth. Forest Ecology and Management, 97(2), 165-72.

33. Leopold D.J., Wali M.K., 1992. The rehabilitation of forest ecosystems in the eastern United States and Canada. Ecosystem Rehabilitation, 2, 187-231.

34. Margalef R. 1958. Information theory in ecology. General Systems, 3, 36-71.

35. Mensah A.K. 2015. Role of revegetation in restoring fertility of degraded mined soils in Ghana: A review. International Journal of Biodiversity and Conservation, 7(2), 57-80.

36. Misra R. 1968. Ecology Work Book. Oxford \& I.B.H. Publishing Co., New Delhi.

37. Mudrák O., Frouz J., Velichová V. 2010. Understory vegetation in reclaimed and unreclaimed postmining forest stands. Ecological Engineering, 36(6), 783-90.

38. Mukhopadhyay S., Maiti S.K., Masto R.E. 2013. Use of reclaimed mine soil index (RMSI) for screening of tree species for reclamation of coal mine degraded land. Ecological Engineering, 57, 133-142.

39. Parrotta J.A. 1992. The role of plantation forests in rehabilitating degraded tropical ecosystems. Agriculture, Ecosystems \& Environment, 41, 115-133.

40. Parrotta J.A. 1999. Productivity, nutrient cycling, and succession in single and mixed-species plantations of Casuarina equisetifolia, Eucalyptus robusta, and Leucaena leucocephala in Puerto Rico. Forest Ecology and Management, 124(1), 45-77.

41. Pensa M., Karu H., Luud A., Rull E., Vaht R. 2008. The effect of planted tree species on the development of herbaceous vegetation in a reclaimed opencast. Canadian Journal of Forest Research, 38(10), 2674-86.

42. Pensa M., Sellin A., Luud A., Valgma I. 2004. An analysis of vegetation restoration on opencast oil shale mines in Estonia. Restoration Ecology, 12(2), 200-206. 
43. Rawlik M., Kasprowicz M., Jagodziński A.M. 2018. Differentiation of herb layer vascular flora in reclaimed areas depends on the species composition of forest stands. Forest Ecology and Management, 409, 541-51.

44. Ryan M.G., Binkley D., Fownes J.H. 1997. Agerelated decline in forest productivity: Pattern and process. Advances in Ecological Research, 27, 213-262.

45. Singh A. 2004. Herbaceous biomass yield on an age series of naturally revegetated mine spoils in a dry tropical environment. Journal of Indian Institute of Science, 84, 53-56.

46. Singh A. 2006. Herbaceous species composition of an age series of naturally revegetated coal mine spoils on Singrauli Coalfields, India. Journal of Indian Institute of Science, 86, 75-79.

47. Singh A. 2012. Pioneer flora on naturally revegetated coal mine spoil in a dry tropical environment. Bulletin of Environment, Pharmacology and Life Sciences, 1(3), 72-73.

48. Singh A. N., Raghubansh A.S., Singh J.S. 2002. Plantations as a tool for mine spoil restoration. Current Science, 82(12), 1436-41.

49. Singh A.N., Raghubanshi A.S., Singh J.S. 2004a. Impact of native tree plantations on mine spoil in a dry tropical environment. Forest Ecology and Management, 187, 49-60.

50. Singh A.N., Raghubanshi A.S., Singh J.S. 2004b. Comparative performance and restoration potential of two Albizia species planted on mine spoil in a dry tropical region, India. Ecological Engineering, 22, 123-140.

51. Singh A.N., Singh J.S. 1999. Biomass net primary production and impact of bamboo plantation on soil redevelopment in a dry tropical region. Forest Ecology and Management, 119, 195-207.

52. Singh A.N., Singh J.S. 2006. Experiments of ecological restoration of coalmine spoil using tree plantation in a dry tropical environment, India: A synthesis. New Forests, 31, 25-39.

53. Singh A.N., Zeng D.H., Chen F.S. 2006. Effect of young woody plantations on Carbon and nutrient accumulation rates in redeveloping soil of coalmine spoil in a dry tropical environment, India. Land Degradation \& Development, 17, 13-21.

54. Singh A.N. 1999. Structure, functioning and impact of young plantations of four native woody species on coal mine spoil. Ph.D. Thesis, Banaras Hindu University, India.

55. Singh J.S., Singh K.P., Jha A.K. 1995. An Integrated Ecological Study on Revegetation of Mine Spoil: Concepts and Research Highlights. Interim Report of an S \& T project sponsored by the Ministry of
Coal, Govt. of India. Department of Botany, Banaras Hindu University, Varanasi.

56. Singh J.S., Yadava P.S. 1974. Seasonal variation in composition, plant biomass, and net primary productivity of a tropical grassland at Kurukshetra, India. Ecological Monographs, 44(3), 351-76.

57. Singh R.S., Singh R.K., Bera S. 2017. Impact of mining on herbaceous ground cover and wildfauna in Birsha-Damoh forest range of Malanjkhand copper mines of India. Eurasian Journal of Forest Science, 5(1), 1-7.

58. Sinha S., Masto R.E., Ram L.C., Selvi V.A., Srivastava N.K., Tripathi R.C., George J. 2009. Rhizosphere soil microbial index of tree species in a coal mining ecosystem. Soil Biology and Biochemistry, 41(9), 1824-32.

59. Spies T.A., Franklin J.F. 1991. The structure of natural young mature and old-growth Douglas-fir forests in Oregon and Washington. In: Ruggiero L.F., Aubry K.B., Carey A.B., Huff M.H. (eds.) Wildlife and Vegetation of Unmanaged Douglas-fir Forests. Portland: US Forest Service General Technical Report, 91-110.

60. Tripathi N., Singh R.S., Hills C.D. 2016. Soil carbon development in rejuvenated Indian coal mine spoil. Ecological engineering, 90, 482-90.

61. Tripathi N., Singh R.S. 2008. Ecological restoration of mined-out areas of dry tropical environment, India. Environmental Monitoring and Assessment, 146(1-3), 325-37.

62. Wade G.L., Thompson R.L. 1993. Species richness on five partially reclaimed Kentucky surface mines. Proc. 10th annual national meeting of the American society for surface mining and reclamation, Spokane.307-314.

63. Wali M.K. 1999. Ecological succession and the rehabilitation of disturbed terrestrial ecosystems. Plant and Soil, 213, 195-220.

64. Whittaker R.H. 1972. Evolution and measurement of species diversity. Taxon, 21, 213-251.

65. Wilson J.B. 1988. Shoot competition and root competition. Journal of Applied Ecology, 25, 279-296.

66. Wiryono W., Siahaan A.B. 2013. Species composition of understory vegetation in coal mined land in central Bengkulu, Indonesia. Biodiversitas, 14 (1), 31-36.

67. Woch M.W., Radwańska M., Stefanowicz A.M. 2013. Flora of spoil heaps after hard coal mining in Trzebinia (Southern Poland): effect of substratum properties. Acta Botanica Croatica, 72(2), 237-56.

68. Woźniak G. 2010. Diversity of vegetation on coalmine heaps of the Upper Silesia (Poland). Szafer Institute of Botany, Polish Academy of Sciences, Kraków, p 310 (in Polish). 